\title{
Cansancio emocional en estudiantes universitarios peruanos en el contexto de la pandemia de Covid-19
}

\author{
Edwin Gustavo Estrada Araozita \\ Universidad Nacional Amazónica de Madre de Dios, Puerto Maldonado, Perú \\ Néstor Antonio Gallegos Ramosiito \\ Universidad Nacional Amazónica de Madre de Dios, Puerto Maldonado, Perú
}

\begin{abstract}
Resumen
La presente investigación tuvo como objetivo describir el cansancio emocional de los estudiantes universitarios peruanos de la carrera profesional de Educación en el contexto de la pandemia de Covid-19. El enfoque fue cuantitativo; el diseño, no experimental; y el tipo, descriptivo transversal. La muestra fue conformada por 232 estudiantes, a quienes se les aplicó la Escala de Cansancio Emocional (2007), instrumento con adecuados niveles de confiabilidad y validez. Según los resultados, la mayoría de estudiantes presentaba altos niveles de cansancio emocional y se pudo determinar que la prevalencia de dicha variable se asociaba de manera significativa al sexo y grupo etario de los estudiantes $(p<0,05)$. Se concluyó que es necesario que el área de Psicopedagogía de la universidad realice la detección oportuna de los estudiantes que padezcan de cansancio emocional para que recuperen su bienestar psicológico y aplique programas preventivos para disminuir la prevalencia de dicho fenómeno.
\end{abstract}

Palabras clave

cansancio emocional, estudiantes, educación universitaria, Covid-19.

\section{Esgotamento emocional em estudantes universitários peruanos} no contexto da pandemia Covid-19

\begin{abstract}
Resumo
A presente pesquisa teve como objetivo descrever o esgotamento emocional de estudantes universitários peruanos da carreira profissional de Educação no contexto da pandemia de Covid-19. A abordagem foi quantitativa; o desenho, não experimental; e o tipo descritivo, transversal. A amostra foi composta por 232 alunos, aos quais foi aplicada a Escala de Cansaço Emocional (2007), instrumento com níveis adequados de confiabilidade e validade. De acordo com os resultados, a maioria dos estudantes apresentou níveis elevados de esgotamento emocional e verificou-se que a prevalência desta variável foi significativamente associada ao sexo e faixa etária dos alunos $(p<0,05)$. Concluiu-se que é necessário que a área de Psicopedagogia da universidade realize a detecção oportuna dos alunos que sofrem de esgotamento emocional para que recuperem o seu bem-estar psicológico e aplique programas preventivos para reduzir a prevalência desse fenômeno.
\end{abstract}

Palavras-chave

esgotamento emocional, estudantes, formação universitária, Covid-19. 


\title{
Emotional exhaustion in Peruvian university students
} in the context of the Covid-19 pandemic

\begin{abstract}
The present research aimed to describe the emotional exhaustion of Peruvian university students from the professional career of Education in the context of the Covid-19 pandemic. The approach was quantitative, the design was non-experimental, and the type was descriptive, cross-sectional. The sample consisted of 232 students, to whom the Emotional Tiredness Scale (2007) was applied, an instrument with adequate levels of reliability and validity. According to the results, the majority of students presented high levels of emotional fatigue and it was determined that the prevalence of this variable was significantly associated with the sex and age group of the students $(p<0.05)$. It was concluded that it is necessary for the Psychopedagogy area of the university to carry out the timely detection of students who suffer from emotional fatigue so that they recover their psychological well-being and apply preventive programs to reduce the prevalence of this phenomenon.
\end{abstract}

\section{Keywords}

emotional exhaustion, students, university education, Covid-19.

\section{Introducción}

A finales del año 2019, se reportaron en China casos de neumonía atípica asociados a un nuevo coronavirus, al cual se le denominó SARS-CoV-2, que causaba la enfermedad de Covid-19 (MOJICA; MORALES, 2020; QUIROZ et al., 2020). Debido al aumento exponencial de casos en todo el mundo, dicha enfermedad fue catalogada por la Organización Mundial de la Salud (OMS) como pandemia internacional en marzo del año 2020 (CAMPOS; ALVES, 2021; QAZI et al., 2020), situación que puso a prueba a los sistemas sanitarios de todos los países (BUITRAGO et al., 2020). Desde luego, este contexto provocó un impacto significativo en la sociedad debido a las repercusiones sanitarias, sociales, económicas, educativas, culturales, etc. (LIZCANO; ARROYAVE, 2020; MURILLO; DUK, 2020).

En el Perú, la pandemia de Covid-19 provocó que el Gobierno de turno decretara el 15 de marzo del año 2020 el estado de emergencia nacional y el aislamiento social obligatorio como una medida excepcional para evitar que el virus se propagara (MIÑAN et al., 2021). Centrándonos en el ámbito educativo, dicha medida provocó que las actividades académicas de todas las instituciones educativas - básicas y superiores - se interrumpieran (ESTRADA et al., 2020; MEJíA et al., 2021) y que la modalidad de 
enseñanza-aprendizaje migrara de la presencialidad a la virtualidad (CRUZ; TOLEDO; MENDOZA, 2021; ESTRADA; MAMANI, 2021; POSTIGO; ARIAS; RAMOS, 2021). No obstante, esta reforma educativa obligatoria provocó mucha preocupación en los docentes y estudiantes, ya que muchos de ellos no estaban acostumbrados ni preparados para afrontar esta nueva metodología educativa (ESTRADA et al., 2021; SUÁREZ et al., 2021). A pesar de lo expuesto, la prestación del servicio educativo siguió en marcha, puesto que existía la necesidad de que no se viera interrumpido y no afectara a la comunidad educativa (ORDORIKA, 2020; TOQUERO, 2020).

Ahora bien, se sabe que el contexto universitario se caracteriza por las exigencias y demandas académicas, personales y sociales para los estudiantes (BRAND et al., 2014). Sumado a ello y tal como se mencionó, la pandemia hizo más compleja la situación, puesto que ellos tuvieron que lidiar, además, con algunos problemas emergentes, como la accesibilidad y conectividad (MOLINA et al., 2021), el temor a contagiarse de Covid-19 y la precariedad económica (ORELLANA, C.; ORELLANA, L., 2020), factores que elevaron sus niveles de estrés y favorecieron la prevalencia de un fenómeno bastante común en estas épocas: el cansancio emocional.

El cansancio emocional ha sido muy estudiado en el ámbito laboral, no obstante, en la presente investigación, será abordado dentro del entorno académico, ya que no solo lo padecen los trabajadores, sino también los estudiantes, principalmente de educación superior (FÍNEZ; MORÁN, 2014). Según el modelo teórico propuesto por Maslach, Schaufeli y Leiter (2001), el cansancio emocional (provocado por las excesivas demandas académicas), la despersonalización (actitud de indiferencia hacia las actividades académicas) y la insatisfacción al logro (conciencia de ineficacia como estudiante) son los factores que configuran el burnout académico, pero el que más predomina es el cansancio emocional, ya que los otros dos factores mencionados no fueron observados de manera significativa ni recurrente en ellos (BARRETO; SALAZAR, 2021; DOMíNGUEZ, 2013, 2014; LANGADE et al., 2016; LLEDÓ et al., 2014).

El cansancio emocional es conceptualizado como el desgaste de energías, la fatiga y el agotamiento, tanto físico como psicológico, que presentan los estudiantes (VIZOSO; ARIAS, 2018), ocasionado por las excesivas demandas psicológicas, que puede llevar a las personas a sentir que sus recursos internos se están agotando (ALSHARIF, 2020). Se caracteriza por las manifestaciones de síntomas somáticos y 
psicológicos y los sentimientos de que ya no se cuenta con la energía suficiente para desenvolverse y realizar las responsabilidades académicas asignadas. Su prevalencia puede afectar las dimensiones académicas, personales, familiares y sociales de los estudiantes. Al respecto, existen diversas investigaciones que reportaron que puede provocar ansiedad, depresión, sentimientos de autoculpa, rumiación, catastroficación e ideación suicida (CABALLERO; GONZALES; PALACIO, 2015; DOMÍNGUEZ; MERINO, 2018; JIMÉNEZ et al., 2019; YOUSSEF, 2016). Asimismo, encontraron asociaciones inversas con la autoeficacia académica, la autoestima, el compromiso y la inteligencia emocional (DOMíNGUEZ, 2018).

Existen investigaciones realizadas en diversas latitudes que buscaron conocer la prevalencia del cansancio emocional en los estudiantes universitarios y lograron identificar que existía una prevalencia considerable (BARRETO; SALAZAR, 2021; CASTRO et al., 2019; ESTRADA; GALLEGOS; MAMANI, 2021; FERNÁNDEZ; LIÉBANA; MORÁN, 2017; HERRERA; MOHAMED; CEPERO, 2016; LEE et al., 2020; LI et al., 2020; OSORIO; PARRELLO; PRADO, 2020; SALAZAR et al., 2021; SEPERAK; FERNÁNDEZ; DOMÍNGUEZ, 2021). Por otro lado, algunas investigaciones determinaron que los niveles de cansancio emocional se asociaban de manera significativa a algunas variables sociodemográficas, como el sexo (BARRETO; SALAZAR, 2021; DOMíNGUEZ et al., 2018; ESTRADA; GALLEGOS, 2020; FERNÁNDEZ; LIÉBANA; MORÁN, 2017; HERRERA; MOHAMED; CEPERO, 2016; LLEDÓ et al., 2014) y el grupo etario de los estudiantes (AGUAYO et al., 2019; BOLAÑOS; RODRÍGUEZ, 2016; ESTRADA; GALLEGOS, 2020). En ese sentido, eran las mujeres y los estudiantes más jóvenes quienes presentaban mayores niveles de cansancio emocional que los varones y estudiantes de más edad, respectivamente.

El estudio del cansancio emocional y la determinación de su prevalencia es un tema muy importante debido a las implicancias que tiene en la salud física, emocional y en su desenvolvimiento académico. A nivel regional y nacional, son muy pocas las investigaciones que estudiaron dicho fenómeno, por lo que se espera, a partir de los hallazgos, que se diseñen y ejecuten programas preventivos y correctivos con el propósito de disminuir su prevalencia y las secuelas que podría originar en los estudiantes. De esta manera, podrían mejorar su calidad de vida y afrontar eficazmente la vida universitaria. 
Finalmente, el objetivo de la presente investigación fue describir el cansancio emocional de los estudiantes universitarios peruanos de la carrera profesional de Educación en el contexto de la pandemia de Covid-19.

\section{Metodología}

\subsection{Diseño}

La investigación tuvo un abordaje cuantitativo, puesto que, para contrastar las hipótesis, se realizó la recolección de datos, lo que permitió su sistematización y posterior análisis estadístico (SÁNCHEZ; REYES; MEJÍA, 2018). El diseño fue no experimental, ya que la variable cansancio emocional no fue manipulada intencionalmente, sino se observó tal como se dio en su entorno (HERNÁNDEZ; MENDOZA, 2018). Por último, el tipo de investigación fue descriptivo transversal, puesto que se analizaron las propiedades y características de la variable cansancio emocional y la medición se realizó en un solo momento respectivamente (BISQUERRA, 2009).

\subsection{Población y muestra}

La población fue conformada por 586 estudiantes de la carrera profesional de Educación de la Universidad Nacional Amazónica de Madre de Dios, ubicada en la ciudad de Puerto Maldonado, Perú. Por otro lado, la muestra estuvo constituida por 232 estudiantes, cantidad que fue determinada a través de un muestreo probabilístico, con un nivel de confianza del $95 \%$ y un nivel de significancia del $5 \%$. Del total de participantes, el $53 \%$ eran mujeres y el $47 \%$ eran hombres. En cuanto al grupo etario al que pertenecían, el $43,1 \%$ tenían entre 16 y 20 años, el $28 \%$ tenían entre 21 y 25 años, el $19 \%$ tenían entre los 25 y 30 años y el 9,9\% tenían más de 30 años. Respecto a las especialidades a las que pertenecían los estudiantes, el 46,6\% eran de Inicial y Especial, el $35,3 \%$ eran de Primaria e Informática y el $18,1 \%$ pertenecían a Matemática y Computación. Con relación a la condición laboral de los estudiantes, el 47,8\% trabajaban y el $52,2 \%$ no trabajaban. Finalmente, en cuanto al nivel socioeconómico, el 51,7\% 
provenían de un nivel medio, el 38,3\% eran de un nivel bajo y el 9,9 pertenecían a un nivel socioeconómico alto. En la Tabla 1 se observan las características de la muestra.

Tabla 1 - Caracterización de la muestra

\begin{tabular}{llcc}
\hline \multicolumn{2}{c}{ Características sociodemográficas } & $\mathbf{n = 2 3 2}$ & $\%$ \\
\hline \multirow{2}{*}{ Sexo } & Hombre & 109 & 47,0 \\
& Mujer & 123 & 53,0 \\
\hline \multirow{3}{*}{ Grupo etario } & De 16 a 20 años & 100 & 43,1 \\
& De 21 a 25 años & 65 & 28,0 \\
& De 25 a 30 años & 44 & 19,0 \\
& De 30 a más años & 23 & 9,9 \\
\hline \multirow{2}{*}{ Especialidad } & Inicial y Especial & 108 & 46,6 \\
& Primaria e Informática & 82 & 35,3 \\
& Matemática y Computación & 42 & 18,1 \\
\hline \multirow{2}{*}{ Condición laboral } & Trabaja & 111 & 47,8 \\
& No trabaja & 121 & 52,2 \\
\hline \multirow{2}{*}{ Nivel socioeconómico } & Bajo & 89 & 38,4 \\
& Medio & 120 & 51,7 \\
& Alto & 23 & 9,9 \\
\hline
\end{tabular}

Fuente: Elaboración propia (2021).

\subsection{Instrumentos}

El instrumento utilizado para la recolección de datos fue la Escala de Cansancio Emocional (GONZALES; LANDERO, 2007), la cual se caracterizó por ser de factor único. Está constituida por 10 ítems de tipo Likert (nunca, muy raras veces, raras veces, a menudo y siempre) y la puntuación obtenida oscila entre los 10 y los 50 puntos.

Tabla 2 - Estructura de la Escala de Cansancio Emocional

\begin{tabular}{ll}
\hline & \multicolumn{1}{c}{ Ítems } \\
\hline 1. & Los exámenes me producen una tensión excesiva. \\
2. & Creo que me esfuerzo mucho para lo poco que consigo. \\
3. & Me siento bajo de ánimo, como triste, sin motivo aparente. \\
4. & Hay días que no duermo bien a causa del estudio. \\
5. & Tengo dolor de cabeza y otras molestias que afectan a mi rendimiento. \\
6. & Hay días que noto más la fatiga y me falta energía para concentrarme. \\
7. & Me siento emocionalmente agotado(a) por mis estudios. \\
8. & Me siento cansado(a) al final de la jornada de estudios. \\
9. & Pensar en los exámenes me produce estrés. \\
10. & Me falta tiempo y me siento sobrecargado por los estudios.
\end{tabular}

Fuente: Gonzales y Landero (2007).

Educ. Form., Fortaleza, v. 7, n. 1, e6759, jan./abr. 2022

DOI: https://doi.org/10.25053/redufor.v7i1.6759

https://revistas.uece.br/index.php/redufor/index ISSN: 2448-3583 
Sus propiedades psicométricas se determinaron mediante los procedimientos de validez y confiabilidad. La validez de contenido se obtuvo mediante la técnica de juicio de expertos, para lo cual se recurrió a tres especialistas, obteniéndose un coeficiente $V$ de Aiken de 0,902, lo cual indicaba que dicha escala posee una muy adecuada validez de contenido. Respecto a la confiabilidad, fue determinada a través de una prueba piloto realizada a 20 estudiantes, lo que permitió obtener un coeficiente Alfa de Cronbach de 0,911, lo que significó que la escala era altamente confiable.

\subsection{Procedimiento}

La recolección de datos fue realizada en varios momentos. En primer lugar, se contactó y explicó el objetivo del estudio y se pidió el permiso a las autoridades universitarias competentes. Luego, se contactó a los estudiantes a través del uso de la red social digital Whatsapp con el propósito de indicarles la finalidad de la investigación y mandarles el enlace para que accedieran a Google Forms, aplicación donde se estructuró el instrumento. Una vez que lograron acceder al mencionado formulario, leyeron con atención las indicaciones y brindaron su consentimiento informado, admitiendo que su participación se daba de manera voluntaria. Seguidamente, respondieron al cuestionario durante un tiempo aproximada de ocho minutos. El acceso a la escala fue inhabilitado una vez que se obtuvieron las 232 respuestas.

\subsection{Análisis estadístico}

Para efectuar el análisis estadístico, se recurrió al software SPSS versión 22, donde los resultados descriptivos fueron sistematizados en tablas de frecuencia y tablas cruzadas. Del mismo modo, la parte inferencial fue realizada utilizando la prueba no paramétrica Chi Cuadrado $\left(\mathrm{X}^{2}\right)$ con el propósito de conocer si la variable cansancio emocional se asociaba de manera significativa con las demás variables sociodemográficas propuestas. 


\section{Resultados}

Los hallazgos que se muestran a continuación proceden de la recolección de datos realizada a los estudiantes universitarios de la carrera profesional de Educación de una universidad pública peruana.

En la Tabla 3 se observa que el $50,4 \%$ de los estudiantes presentaban altos niveles de cansancio emocional, el 34,5\% tenían niveles moderados y el $15,1 \%$ evidenciaban bajos niveles. Lo expuesto indica que los estudiantes se caracterizaban por presentar un conjunto de sintomatologías somáticas (tensión, insomnio y cefaleas) así como psicológicas (ansiedad, estrés, tensión, frustración), las cuales provocaban la disminución de sus energías para realizar sus actividades académicas y afrontar la vida universitaria.

Tabla 3 - Resultados descriptivos del cansancio emocional

\begin{tabular}{lcc}
\hline Nivel de cansancio emocional & $\mathbf{n}$ & $\%$ \\
\hline Bajo & 35 & 15,1 \\
Moderado & 80 & 34,5 \\
Alto & 117 & 50,4 \\
Total & 232 & 100,0 \\
\hline
\end{tabular}

Fuente: Elaboración propia (2021).

En la Tabla 4 se observa que el sexo de los estudiantes se asoció de manera significativa con el nivel de cansancio emocional $(p<0,05)$. Así pues, se ve que las mujeres presentaron mayores niveles de cansancio emocional que los hombres, ya que en ellas predominaba el nivel alto $(60,1 \%)$, mientras que en ellos, el nivel moderado $(41,3 \%)$.

Tabla 4 - Niveles de cansancio emocional según el sexo de los estudiantes

\begin{tabular}{ccccccc}
\hline \multirow{2}{*}{$\begin{array}{c}\text { Variable } \\
\text { sociodemográfica }\end{array}$} & \multicolumn{3}{c}{ Cansancio emocional } & \multirow{2}{*}{$\mathrm{X}^{2}$} & \multirow{2}{*}{ Sig. } \\
\cline { 2 - 5 } & $\mathrm{n}(\%)$ & $\begin{array}{c}\text { Moderado } \\
\mathrm{n}(\%)\end{array}$ & $\begin{array}{c}\text { Alto } \\
\mathrm{n}(\%)\end{array}$ & & \\
\hline \multirow{2}{*}{ Sexo } & Hombre & $21(19,3)$ & $45(41,3)$ & $43(39,4)$ & 21,432 & 0 \\
& Mujer & $14(11,4)$ & $35(28,5)$ & $74(60,1)$ & 200 \\
\hline
\end{tabular}

Fuente: Elaboración propia (2021).

Según la Tabla 5, el grupo etario al cual pertenecían los estudiantes se asoció significativamente con el nivel de cansancio emocional $(p<0,05)$. En virtud de lo expuesto, se observa que los estudiantes más jóvenes presentaron mayores niveles de 
cansancio emocional que los estudiantes de mayor edad, ya que en los primeros predominaba el nivel alto, mientras que en los últimos, el nivel moderado.

Tabla 5 - Niveles de cansancio emocional según el grupo etario de los estudiantes

\begin{tabular}{|c|c|c|c|c|c|c|}
\hline \multirow{2}{*}{\multicolumn{2}{|c|}{ Variable sociodemográfica }} & \multicolumn{3}{|c|}{ Cansancio emocional } & \multirow[b]{2}{*}{$x^{2}$} & \multirow[b]{2}{*}{ Sig. } \\
\hline & & $\begin{array}{l}\text { Bajo } \\
\text { n (\%) }\end{array}$ & $\begin{array}{c}\text { Moderado } \\
\mathrm{n}(\%)\end{array}$ & $\begin{array}{c}\text { Alto } \\
\mathrm{n}(\%)\end{array}$ & & \\
\hline \multirow{4}{*}{$\begin{array}{l}\text { Grupo } \\
\text { etario }\end{array}$} & De 16 a 20 años & $10(10,0)$ & $30(30,0)$ & $60(60,0)$ & \multirow{4}{*}{7,943} & \multirow{4}{*}{0,002} \\
\hline & De 21 a 25 años & $7(10,8)$ & $22(33,8)$ & $36(55,4)$ & & \\
\hline & De 25 a 30 años & $10(22,7)$ & $19(43,2)$ & $15(34,1)$ & & \\
\hline & De 30 a más años & $8(34,8)$ & $9(39,1)$ & $6(26,1)$ & & \\
\hline
\end{tabular}

Fuente: Elaboración propia (2021).

De acuerdo con la Tabla 6, la especialidad a la cual pertenecían los estudiantes no se asoció significativamente con el nivel de cansancio emocional ( $p>0,05)$. Por ello, se puede ver que los estudiantes de las tres especialidades mostraron un alto nivel de cansancio emocional.

Tabla 6 - Niveles de cansancio emocional según la especialidad de los estudiantes

\begin{tabular}{llccccc}
\hline \multirow{2}{*}{ Variable sociodemográfica } & \multicolumn{3}{c}{ Cansancio emocional } & \multirow{2}{*}{ Sig. } \\
\cline { 2 - 4 } & Bajo & Moderado & Alto & & X & Sig. \\
& Inicial y Especial & $16(14,8)$ & $30(27,8)$ & $62(57,4)$ & & \\
\hline \multirow{2}{*}{ Especialidad } & Primaria e Informática & $10(12,2)$ & $35(42,7)$ & $37(45,1)$ & 9,242 & 0,056 \\
& Matemática y Computación & $9(21,4)$ & $15(35,7)$ & $18(42,9)$ & & \\
\hline
\end{tabular}

Fuente: Elaboración propia (2021).

En la Tabla 7 se observa que la condición laboral de los estudiantes tampoco se asociaba significativamente con el nivel de cansancio emocional $(p>0,05)$. En ese entender, se puede ver que los estudiantes que trabajaban y que no trabajaban presentaron altos niveles de cansancio emocional.

Tabla 7 - Niveles de cansancio emocional según la condición laboral de los estudiantes

\begin{tabular}{|c|c|c|c|c|c|c|}
\hline \multirow{2}{*}{\multicolumn{2}{|c|}{ Variable Sociodemográfica }} & \multicolumn{3}{|c|}{ Cansancio Emocional } & \multirow[b]{2}{*}{$x^{2}$} & \multirow[b]{2}{*}{ Sig. } \\
\hline & & $\begin{array}{c}\text { Bajo } \\
\text { n (\%) }\end{array}$ & $\begin{array}{c}\text { Moderado } \\
\mathrm{n}(\%)\end{array}$ & $\begin{array}{c}\text { Alto } \\
\mathrm{n}(\%)\end{array}$ & & \\
\hline $\begin{array}{c}\text { Condición } \\
\text { laboral }\end{array}$ & $\begin{array}{l}\text { Trabaja } \\
\text { No trabaja }\end{array}$ & $\begin{array}{l}14(12,6) \\
21(17,4)\end{array}$ & $\begin{array}{l}35(31,5) \\
45(37,2)\end{array}$ & $\begin{array}{l}62(55,9) \\
55(45,4)\end{array}$ & 21,301 & 0,069 \\
\hline
\end{tabular}

Fuente: Elaboración propia (2021).

Finalmente, en la Tabla 8 se observa que el nivel socioeconómico de los estudiantes no se asoció de manera significativa con los niveles de cansancio emocional 
$(p>0,05)$. Así pues, se ve que los estudiantes de los niveles bajo, medio y alto presentaron altos niveles de cansancio emocional.

Tabla 8 - Niveles de cansancio emocional según el nivel socioeconómico de los estudiantes

\begin{tabular}{ccccccc}
\hline \multirow{2}{*}{ Variable sociodemográfica } & \multicolumn{3}{c}{ Cansancio emocional } & \multirow{2}{*}{$\mathrm{X}^{2}$} & \multirow{2}{*}{ Sig. } \\
\cline { 2 - 5 } & & $\begin{array}{c}\text { Bajo } \\
\mathrm{n}(\%)\end{array}$ & $\begin{array}{c}\text { Moderado } \\
\mathrm{n}(\%)\end{array}$ & $\begin{array}{c}\text { Alto } \\
\mathrm{n}(\%)\end{array}$ & & \\
\hline \multirow{2}{*}{ Nivel } & Bajo & $11(12,4)$ & $30(33,7)$ & $48(53,9)$ & & \\
\multirow{2}{*}{ socioeconómico } & Medio & $18(15,0)$ & $42(35,0)$ & $60(50,0)$ & \multirow{2}{*}{15,695} & 0,051 \\
& Alto & $6(26,1)$ & $8(34,8)$ & $9(39,1)$ & & \\
\hline
\end{tabular}

Fuente: Elaboración propia (2021).

\section{Discusión}

El cansancio emocional es una faceta fundamental del burnout. Ocurre cuando las demandas académicas exceden los recursos y provoca un agotamiento de energía. Las personas que están emocionalmente cansadas se sienten psicológica y emocionalmente agotadas y pueden experimentar fatiga física.

En primer lugar, se determinó que la mayoría de los estudiantes universitarios presentaron altos niveles de cansancio emocional, lo cual indica que manifestaban una serie de sintomatologías somáticas (tensión, insomnio y cefaleas), así como psicológicas (ansiedad, estrés, tensión, frustración).

La información descrita sería causada por las exigencias y demandas académicas (exámenes, tareas, informes, exposiciones, etc.) y adicionalmente por los problemas emergentes a la educación virtual en el contexto de la pandemia, tales como la hiperconexión y el tecnoestrés, que son producto de la excesiva exposición a las laptops, tablets y celulares que los estudiantes utilizan para participar de las videoconferencias y desarrollar sus trabajos. Del mismo modo, constantemente se suscitan problemas de intermitencia de la señal de internet debido a que muchos de ellos se encuentran en zonas rurales, donde la conectividad es pésima. Ahora bien, también existen problemas ocasionados directamente por la pandemia, como el temor generalizado a contagiarse de Covid-19, y, por otro lado, varios estudiantes tenían serias limitaciones económicas debido al desempleo, ya que sus centros de trabajo fueron cerrados por las medidas restrictivas impuestas por el gobierno. Esta situación provocaría, a su vez, la disminución de sus energías para que realicen sus actividades 
académicas y afronten la vida universitaria, afectando negativamente su bienestar emocional y calidad de vida.

El hallazgo expuesto coincide con lo reportado por Barreto y Salazar (2021), quienes realizaron una investigación en Colombia con el propósito de analizar el agotamiento emocional en los estudiantes de educación superior del área de Salud y determinaron que la prevalencia era alta y se caracterizaba por la presencia de síntomas somáticos (afecciones gástricas, dolores de cabeza, alteraciones en el patrón de sueño) y psicológicos (intranquilidad, tensión, poca concentración, angustia) que experimentaban durante los periodos académicos.

Asimismo, guarda relación con los hallazgos de Osorio, Parrello y Prado (2020), que tenían como propósito determinar los niveles y tipos de burnout académico (cansancio emocional, cinismo e inadecuación) así como su asociación con algunas variables sociodemográficas en estudiantes de una universidad estatal mexicana. Entre sus principales conclusiones, destacan que la mayoría de estudiantes alcanzaron puntajes de cansancio emocional entre medios y altos, lo cual significaba que sentían que estaban exhaustos por cumplir con las responsabilidades académicas y que no podían más con las tareas como estudiantes.

Del mismo modo, es similar al estudio de Lee et al. (2020), quienes realizaron una investigación acerca del cansancio emocional en estudiantes y determinaron que existía una prevalencia significativa de dicho fenómeno; es decir, se encontraban emocionalmente agotados por las sobrecargadas demandas académicas y la falta de recursos para afrontarlas. Ello provocaba una falta de energía, fatiga crónica, apatía, inquietud, nerviosismo y sensación de agotamiento y frustración.

El cansancio emocional se configura como una respuesta básica al estrés (MASLACH, 2003), parte del desequilibrio que existe entre las demandas del contexto académico y los recursos con los que cuenta el estudiante (PRADA; NAVARRO; DOMÍNGUEZ, 2020) y tiene repercusiones negativas, pues influye en las expectativas de éxito y la madurez profesional, produce bajas expectativas de concluir los estudios, tendencia a la deserción y poca preparación para afrontar el mundo laboral (FONTANA, 2011). Por ello, es importante conocer la incidencia del cansancio emocional en los estudiantes, y más aún durante estos tiempos de pandemia, para poder realizar programas de intervención sobre los casos que lo requieran (DOMíNGUEZ et al., 2018). 
Respecto a las variables sociodemográficas, se determinó que el sexo de los estudiantes se asoció de manera significativa con el nivel de cansancio emocional $(p<0,05)$. En ese sentido, las mujeres presentaron mayores niveles de cansancio emocional que los hombres. Lo expuesto podría ser explicado debido a que ellas suelen exteriorizar las manifestaciones emocionales y fisiológicas producidas por situaciones estresantes (ESTRADA et al., 2020). Asimismo, normalmente ellas asumen responsabilidades en sus familias, como el cuidado de los hijos y actividades domésticas (ARIAS; HUAMANí; CEBALLOS, 2019). El hallazgo descrito coincide con algunas investigaciones que determinaron que existía una mayor prevalencia del cansancio emocional en las mujeres que en los varones (BARRETO; SALAZAR, 2021; DOMÍNGUEZ et al., 2018; ESTRADA; GALLEGOS, 2020; FERNÁNDEZ; LIÉBANA; MORÁN et al., 2017; HERRERA; MOHAMED; CEPERO, 2016; LLEDÓ et al., 2014).

Otro hallazgo importante da cuenta que el grupo etario al cual pertenecían los estudiantes también se asoció significativamente con el nivel de cansancio emocional $(p<0,05)$. En virtud de lo expuesto, se observa que los estudiantes más jóvenes presentaron mayores niveles de cansancio emocional que los estudiantes de mayor edad. Ello se debería a que los estudiantes más jóvenes recién estarían aprendiendo a utilizar recursos y estrategias de autorregulación de sus emociones para enfrentar los diversos factores estresantes propios de las actividades académicas universitarias, no obstante, los estudiantes con más edad ya habrían desarrollado, de manera formal o informal, dichos recursos y estrategias. El resultado descrito es similar a los hallazgos de algunos estudios que también encontraron que los estudiantes pertenecientes a los grupos etarios de más edad presentaban niveles de cansancio emocional ligeramente inferiores que los estudiantes más jóvenes (AGUAYO et al., 2019; BOLAÑOS; RODRÍGUEZ, 2016; ESTRADA; GALLEGOS, 2020).

Entre las principales limitaciones de la presente investigación, están la cantidad de estudiantes y su homogeneidad, lo cual no permite generalizar los resultados. Por ello, se recomienda que los futuros estudios incrementen la cantidad de participantes e incluyan a estudiantes de las demás carreras profesionales de la universidad focalizada en el presente estudio para que los resultados puedan generalizarse. 


\section{Consideraciones finales}

El cansancio emocional es un tipo de respuesta frente a las situaciones estresantes que se presentan en los diferentes contextos y es caracterizada por la pérdida de las energías, los sentimientos de desgaste emocional y físico y provocaría que los estudiantes que padezcan de dicha enfermedad incumplan sus responsabilidades académicas y afecten su calidad de vida así como su bienestar psicológico. En ese sentido, se halló que la mayoría de los estudiantes presentaban altos niveles de cansancio emocional, causado principalmente por la hiperconexión, el tecnoestrés, los problemas de conectividad, el temor a contagiarse de Covid-19 y la precariedad económica por la que atravesaban. Asimismo, se pudo determinar que la prevalencia de dicha variable se asociaba de manera significativa al sexo y grupo etario de los estudiantes $(\mathrm{p}<0,05)$. Por ello, es imperativo que el área de Psicopedagogía de la universidad realice la detección oportuna de los estudiantes que padezcan de cansancio emocional para que recuperen su bienestar psicológico y aplique programas preventivos para disminuir la prevalencia de dicho fenómeno.

\section{Referencias}

AGUAYO, R. et al. A risk profile of socio-demographic factors in the onset of academic burnout syndrome in a sample of university students. International Journal of Environmental Research and Public Health, Basilea, v. 16, n. 5, p. 707-716, 2019. DOI: https://doi.org/10.3390/ijerph16050707. Disponible en: https://www.mdpi.com/16604601/16/5/707. Accedido el: 8 ago. 2021.

ALSHARIF, A. The protective role of resilience in emotional exhaustion among dental students at clinical levels. Psychology Research and Behavior Management, Macclesfield, v. 13, p. 989-995, 2020. DOI: https://doi.org/10.2147/prbm.S281580. Disponible en: https://www.dovepress.com/the-protective-role-of-resilience-in-emotionalexhaustion-among-dental-peer-reviewed-fulltext-article-prbm. Accedido el: 6 ago. 2021.

ARIAS, W.; HUAMANÍ, J.; CEBALLOS, K. Síndrome de Burnout en profesores de escuela y universidad: un análisis psicométrico y comparativo en la ciudad de Arequipa. Propósitos y Representaciones, Lima, v. 7, n. 3, p. 72-91, 2019. DOI: http://dx.doi.org/10.20511/pyr2019.v7n3.390. Disponible en: http://revistas.usil.edu.pe/index.php/pyr/article/view/390. Accedido el: 15 set. 2021. 
BARRETO, D.; SALAZAR, H. Agotamiento emocional en estudiantes universitarios del área de la salud. Universidad y Salud, San Juan de Pasto, v. 23, n. 1, p. 30-39, 2021. DOI: https://doi.org/10.22267/rus.212301.211. Disponible en:

https://revistas.udenar.edu.co/index.php/usalud/article/view/4644. Accedido el: 6 ago. 2021.

BISQUERRA, R. Metodología de la investigación educativa. 2. ed. Madrid: La Muralla, 2009.

BOLAÑOS, N.; RODRÍGUEZ, N. Prevalencia del Síndrome de Burnout académico en el estudiantado de Enfermería de la Universidad de Costa Rica. Revista Enfermería Actual en Costa Rica, San José, v. 31, p. 1-19, 2016. DOI:

http://dx.doi.org/10.15517/revenf.v0i31.24519. Disponible en:

https://revistas.ucr.ac.cr/index.php/enfermeria/article/view/24519. Accedido el: 8 ago. 2021.

BRAND, S. et al. Adolescents with greater mental toughness show higher sleep efficiency, more deep sleep and fewer awakenings after sleep onset. The Journal of Adolescent Health, New York, v. 54, n. 1, p. 109-113, 2014. DOI:

https://doi.org/10.1016/j.jadohealth.2013.07.017. Disponible en:

https://www.jahonline.org/article/S1054-139X(13)00382-0/fulltext. Accedido el: 6 ago. 2021.

BUITRAGO, F. et al. Pandemia de la Covid-19 y salud mental: reflexiones iniciales desde la atención primaria de salud española. Atención Primaria, Barcelona, v. 53, n. 1, p. 89101, 2021. DOI: https://doi.org/10.1016/j.aprim.2020.06.006. Disponible en: https://www.sciencedirect.com/science/article/pii/S0212656720301876?via\%3Dihub. Accedido el: 6 ago. 2021.

CABALLERO, C.; GONZALES, O.; PALACIO, J. Relación del burnout y el engagement con depresión, ansiedad y rendimiento académico en estudiantes universitarios. Revista Científica Salud Uninorte, Barranquilla, v. 31, n. 1, p. 59-69, 2015. DOI:

http://dx.doi.org/10.14482/sun.31.1.5085. Disponible en: https://rcientificas.uninorte.edu.co/index.php/salud/article/view/5085. Accedido el: 6 ago. 2021.

CAMPOS, J.; ALVES, L. Percepções sobre as ações das redes públicas de ensino durante a pandemia. Educação \& Formação, Fortaleza, v. 6, n. 3, e5412, 2021. DOI: https://doi.org/10.25053/redufor.v6i3.5412. Disponible en:

https://revistas.uece.br/index.php/redufor/article/view/5412. Accedido el: 15 set. 2021.

CASTRO, Y. et al. Agotamiento emocional en estudiantes de Odontología de la Universidad Nacional Mayor de San Marcos. Revista Habanera de Ciencias Médicas, La Habana, v. 18, n. 1, p. 150-163, 2019. Disponible en:

http://www.revhabanera.sld.cu/index.php/rhab/article/view/2427. Accedido el: 8 ago. 2021.

CRUZ, L.; TOLEDO, E.; MENDOZA, A. Qualidade do serviço educativo na Faculdade de Ciências Sociais e Humanas de uma universidade pública. Educação \& Formação, Fortaleza, v. 6, n. 3, e5293, 2021. DOI: https://doi.org/10.25053/redufor.v6i3.5293. Disponible en: https://revistas.uece.br/index.php/redufor/article/view/5293. Accedido el: 15 de set. 2021. 
DOMíNGUEZ, S. Agotamiento emocional académico en estudiantes universitarios: ¿Cuánto influyen las estrategias cognitivas de regulación emocional? Educación Médica, Murcia, v. 19, n. 2, p. 96-103, 2018. DOI: https://doi.org/10.1016/j.edumed.2016.11.010. Disponible en:

https://www.sciencedirect.com/science/article/pii/S1575181316301607?via\%3dihub. Accedido el: 6 ago. 2021.

DOMÍNGUEZ, S. Análisis psicométrico de la Escala de Cansancio Emocional en estudiantes de una universidad privada. Revista Digital de Investigación en Docencia Universitaria, Lima, v. 7, n. 1, p. 45-55, 2013. DOI: https://doi.org/10.19083/ridu.7.186. Disponible en: https://revistas.upc.edu.pe/index.php/docencia/article/view/186. Accedido el: 6 ago. 2021.

DOMÍNGUEZ, S. Escala de Cansancio Emocional: estructura factorial y validez de los ítems en estudiantes de una universidad privada. Avances en Psicología, Lima, v. 22, n. 1, p. 89-97, 2014. DOI: https://doi.org/10.33539/avpsicol.2014.v22n1.275. Disponible en: https://revistas.unife.edu.pe/index.php/avancesenpsicologia/article/view/275.

Accedido el: 6 ago. 2021.

DOMÍNGUEZ, S. et al. Datos normativos de una escala de agotamiento emocional académico en estudiantes universitarios de psicología de Lima (Perú). Educación Médica, Murcia, v. 19, n. S3, p. 246-255, 2018. DOI: https://doi.org/10.1016/j.edumed.2017.09.002. Disponible en: https://www.sciencedirect.com/science/article/pii/S1575181317301705?via\%3Dihub. Accedido el: 8 ago. 2021.

DOMÍNGUEZ, S.; MERINO, C. Cognitive emotional regulation questionnaire-18 en universitarios: evidencias de validez convergente y discriminante. Revista Iberoamericana de Diagnóstico y Evaluación - e Avaliação Psicológica, Lisboa, v. 47, n. 2, p. 171-184, 2018. DOI: https://doi.org/10.21865/ridep47.2.12. Disponible en: https://dialnet.unirioja.es/servlet/articulo?codigo=6483787. Accedido el: 6 ago. 2021.

ESTRADA, E.; GALLEGOS, N. Síndrome de Burnout y variables sociodemográficas en docentes peruanos. Archivos Venezolanos de Farmacología y Terapéutica, Caracas, v. 39, n. 6, p. 714-720, 2020. DOI: http://doi.org/10.5281/zenodo.4404750. Disponible en: http://190.169.30.98/ojs/index.php/rev aavft/article/view/21074/144814487374. Accedido el: 8 ago. 2021.

ESTRADA, E.; GALLEGOS, N.; MAMANI, H. Burnout académico en estudiantes universitarios peruanos. Apuntes Universitarios, Lima, v. 11, n. 2, p. 48-62, 2021. DOI: https://doi.org/10.17162/au.v11i2.631. Disponible en:

https://apuntesuniversitarios.upeu.edu.pe/index.php/revapuntes/article/view/631. Accedido el: 8 ago. 2021.

ESTRADA, E.; MAMANI, M. Competencia digital y variables sociodemográficas en docentes peruanos de educación básica regular. Revista San Gregorio, Manabí, v. 1, 
n. 45, p. 1-16, 2021. DOI: http://dx.doi.org/10.36097/rsan.v0i45.1502. Disponible en: http://revista.sangregorio.edu.ec/index.php/revistasangregorio/article/view/1502.

Accedido el: 6 ago. 2021.

ESTRADA, E. et al. Actitud de los estudiantes universitarios frente a la educación virtual en tiempos de la pandemia de Covid-19. Revista Brasileira de Educação do Campo, Tocantins, v. 5, e10237, 2020. DOI: https://doi.org/10.20873/uft.rbec.e10237. Disponible en: https://sistemas.uft.edu.br/periodicos/index.php/campo/article/view/10237. Accedido el: 6 ago. 2021.

ESTRADA, E. et al. Estrés académico en estudiantes universitarios peruanos en tiempos de la pandemia del Covid-19. Archivos Venezolanos de Farmacología y Terapéutica, Caracas, v. 40, n. 1, p. 88-93, 2021. DOI: http://doi.org/10.5281/zenodo.4675923.

Disponible en: http://saber.ucv.ve/ojs/index.php/rev aavft/article/view/22351. Accedido el: 6 ago. 2021.

FERNÁNDEZ, E.; LIÉBANA, C.; MORÁN, C. Relación entre el sentido de coherencia y el cansancio emocional en estudiantes universitarios. Psychology, Society, \& Education, Almería, v. 9, n. 3, p. 393-403, 2017. DOI: http://dx.doi.org/10.25115/psye.v9i3.861. Disponible en: http://ojs.ual.es/ojs/index.php/psye/article/view/861. Accedido el: 8 ago. 2021.

FÍNEZ, M.; MORÁN, C. Resiliencia y autoconcepto: su relación con el cansancio emocional en adolescentes. INFAD Revista de Psicología, Badajoz, v. 6, n. 1, p. 289296, 2014. DOI: https://doi.org/10.17060/ijodaep.2014.n1.v6.746. Disponible en: https://revista.infad.eu/index.php/lJODAEP/article/view/746. Accedido el: 6 ago. 2021.

FONTANA, S. Estudio preliminar de las propiedades psicométricas de la escala de desgaste emocional para estudiantes universitarios. Revista Argentina de Ciencias del Comportamiento, Córdoba, v. 3, n. 2, p. 44-48, 2011. DOI: https://doi.org/10.32348/1852.4206.v3.n2.5227. Disponible en: https://revistas.unc.edu.ar/index.php/racc/article/view/5227. Accedido el: 8 ago. 2021.

GONZALES, M.; LANDERO, R. Escala de Cansancio Emocional (ECE) para estudiantes universitarios: propiedades psicométricas en una muestra de México. Anales de Psicología, Murcia, v. 23, n. 2, p. 253-257, 2007. Disponible en: https://revistas.um.es/analesps/article/view/22321. Accedido el: 8 ago. 2021.

HERNÁNDEZ, R.; MENDOZA, C. Metodología de la investigación: las rutas cuantitativa, cualitativa y mixta. México: McGraw-Hill, 2018.

HERRERA, L.; MOHAMED, L.; CEPERO, S. Cansancio emocional en estudiantes universitarios. Dedica: Revista de Educação e Humanidades, Granada, v. 9, p. 173-191, 2016. DOI: https://doi.org/10.30827/dreh.v0i9.6888. Disponible en: https://revistaseug.ugr.es/index.php/dedica/article/view/6888. Accedido el: 8 ago. 2021.

JIMÉNEZ, J. et al. Emotional exhaustion, burnout, and perceived stress in dental students. Journal of International Medical Research, London, v. 47, n. 9, p. 4251-4259, 
2019. DOI: https://doi.org/10.1177/0300060519859145. Disponible en:

https://journals.sagepub.com/doi/10.1177/0300060519859145. Accedido el: 6 ago. 2021.

LANGADE, D. et al. Burnout syndrome among medical practitioners across India: A Questionnaire-Based Survey. Cureus, United States, v. 8, n. 9, e771, 2016. DOI: https://doi.org/10.7759/cureus.771. Disponible en: https://www.cureus.com/articles/4941burnout-syndrome-among-medical-practitioners-across-india-a-questionnaire-basedsurvey. Accedido el: 6 ago. 2021.

LEE, M. et al. From emotional exhaustion to cynicism in academic burnout among Korean high school students: focusing on the mediation effects of hatred of academic work. Stress \& Health, United States, v. 36, n. 3, p. 376-383, 2020. DOI: https://doi.org/10.1002/smi.2936. Disponible en: https://onlinelibrary.wiley.com/doi/full/10.1002/smi.2936. Accedido el: 8 ago. 2021.

LI, C. et al. Emotional exhaustion and sleep problems in university students: does mental toughness matter?. Personality and Individual Differences, Amsterdam, v. 163, e110046, 2020. DOI: https://doi.org/10.1016/i.paid.2020.110046. Disponible en:

https://www.sciencedirect.com/science/article/abs/pii/S019188692030235X. Accedido el: 8 ago. 2021.

LIZCANO, F.; ARROYAVE, F. El ambiente, los desplazamientos y el riesgo cardiovascular en la pandemia por Covid-19. Revista Colombiana de Cardiología, Bogotá, v. 27, n. 3, p. 160-165, 2020. DOI: https://doi.org/10.1016/i.rccar.2020.05.001. Disponible en: https://www.sciencedirect.com/science/article/pii/S0120563320300905. Accedido el: 6 e ago. 2021.

LLEDÓ, A. et al. Cansancio emocional, autoestima y satisfacción con los estudios en estudiantes universitarios. INFAD Revista de Psicología, Badajoz, v. 7, n. 1, p. 161-170, 2014. DOI: https://doi.org/10.17060/ijodaep.2014.n1.v7.787. Disponible en: https://revista.infad.eu/index.php/ijodaep/article/view/787. Accedido el: 6 ago. 2021.

MASLACH, C. Job burnout: new directions in research and intervention. Current Directions in Psychological Science, United States, v. 12, n. 5, p. 189-192, 2003. DOI: https://doi.org/10.1111/1467-8721.01258. Disponible en: https://journals.sagepub.com/doi/10.1111/1467-8721.01258. Accedido el: 8 ago. 2021 .

MASLACH, C.; SCHAUFELI, W.; LEITER, M. Job burnout. Annual Review of Psychology, California, v. 52, p. 397-422, 2001. DOI: https://doi.org/10.1146/annurev.psych.52.1.397. Disponible en: https://www.annualreviews.org/doi/10.1146/annurev.psych.52.1.397. Accedido el: 6 ago. 2021.

MEJíA, C. et al. Repercusión académica de la Covid-19 en universitarios peruanos. Revista Cubana de Investigaciones Biomédicas, La Habana, v. 40, n. 1, e814, 2021. Disponible en: http://www.revibiomedica.sld.cu/index.php/ibi/article/view/814. Accedido el: 6 ago. 2021. 
MIÑAN, G. et al. La pandemia del Covid-19 en el Perú y la función del ingeniero industrial. Universidad y Sociedad, Cienfuegos, v. 13, n. 1, p. 59-63, 2021. Disponible en: https://rus.ucf.edu.cu/index.php/rus/article/view/1897. Accedido el: 6 ago. 2021.

MOJICA, R.; MORALES, M. Pandemia Covid-19, la nueva emergencia sanitaria de preocupación internacional: una revisión. Medicina de Familia: Semergen, Madrid, v. 46, n. 1, p. 65-77, 2020. DOI: https://doi.org/10.1016/j.semerg.2020.05.010. Disponible en: https://www.sciencedirect.com/science/article/abs/pii/S1138359320301714?via\%3Dihub. Accedido el: 6 ago. 2021.

MOLINA, T. Crisis estudiantil en pandemia. ¿Cómo valoran los estudiantes universitarios la educación virtual?. Revista Conrado, Cienfuegos, v. 17, n. 80, p. 283-294, 2021. Disponible en: https://conrado.ucf.edu.cu/index.php/conrado/article/view/1845. Accedido el: 6 ago. 2021.

MURILLO, F.; DUK, C. El Covid-19 y las brechas educativas. Revista Latinoamericana de Educación Inclusiva, Santiago de Chile, v. 14, n. 1, p. 11-13, 2020. DOI: http://dx.doi.org/10.4067/S0718-73782020000100011. Disponible en: https://www.scielo.cl/scielo.php?script=sci arttext\&pid=S071873782020000100011\&lng=en\&nrm=iso\&tlng=en. Accedido el: 6 ago. 2021.

ORDORIKA, I. Pandemia y educación superior. Revista de la Educación Superior, Ciudad de México, v. 49, n. 194, p. 1-8, 2020. DOI: https://doi.org/10.36857/resu.2020.194.1120. Disponible en: http://resu.anuies.mx/ojs/index.php/resu/article/view/1120. Accedido el: 6 ago. 2021.

ORELLANA, C.; ORELLANA, L. Predictores de síntomas emocionales durante la cuarentena domiciliar por pandemia de Covid-19 en El Salvador. Actualidades en Psicología, San José, v. 34, n. 128, p. 103-120, 2020. DOI: https://doi.org/10.15517/ap.v34i128.41431. Disponible en: https://revistas.ucr.ac.cr/index.php/actualidades/article/view/41431. Accedido el: 6 ago. 2021.

OSORIO, M.; PARRELLO, S.; PRADO, C. Burnout académico en una muestra de estudiantes universitarios mexicanos. Enseñanza e Investigación en Psicología, Ciudad México, v. 2, n. 1, p. 27-37, 2020. Disponible en: https://www.revistacneip.org/index.php/cneip/article/view/86. Accedido el: 8 ago. 2021.

POSTIGO, J.; ARIAS, D.; RAMOS, T. Percepção de interação em sala de aula virtual e nível de habilidades sociais em estudantes universitários. Educação \& Formação, Fortaleza, v. 6, n. 3, e5297, 2021. DOI: https://doi.org/10.25053/redufor.v6i3.5297. Disponible en: https://revistas.uece.br/index.php/redufor/article/view/5297. Accedido el: 15 set. 2021.

PRADA, R.; NAVARRO, J.; DOMÍNGUEZ, S. Personalidad y agotamiento emocional académico en estudiantes universitarios peruanos: un estudio predictivo. Revista Digital de Investigación en Docencia Universitaria, Lima, v. 14, n. 2, e1227, 2020. DOI: 
https://doi.org/10.19083/ridu.2020.1227. Disponible en:

https://revistas.upc.edu.pe/index.php/docencia/article/view/1227. Accedido el: 8 ago. 2021.

QAZI, A. et al. Conventional to online education during Covid-19 pandemic: do develop and underdeveloped nations cope alike. Children and Youth Services Review, London, v. 119, e105582, 2020. DOI: https://doi.org/10.1016/i.childyouth.2020.105582. Disponible en: https://www.sciencedirect.com/science/article/pii/S0190740920320053?via\%3Dihub. Accedido el: 6 ago. 2021.

QUIROZ, C. et al. Un nuevo coronavirus, una nueva enfermedad: Covid-19. Horizonte Médico, Lima, v. 20, n. 2, e1208, 2020. DOI:

https://doi.org/10.24265/horizmed.2020.v20n2.11. Disponible en:

https://www.horizontemedico.usmp.edu.pe/index.php/horizontemed/article/view/1208. Accedido el: 6 ago. 2021.

SALAZAR, J. et al. Síndrome de burnout en estudiantes de educación superior tecnológica del campus Tierra Blanca en tiempo de covid-19. Revista Iberoamericana para la Investigación y el Desarrollo Educativo, Jalisco, v. 11, n. 22, e204, 2021. DOI: https://doi.org/10.23913/ride.v11i22.896. Disponible en: https://mail.ride.org.mx/index.php/RIDE/article/view/896. Accedido el: 8 ago. 2021.

SÁNCHEZ, H.; REYES, C.; MEJÍA, K. Manual de términos en investigación científica, tecnológica y humanística. Lima: Universidad Ricardo Palma, 2018.

SEPERAK, R.; FERNÁNDEZ, M.; DOMÍNGUEZ, S. Prevalencia y severidad del burnout académico en estudiantes universitarios durante la pandemia por la Covid-19.

Interacciones, Lima, v. 7, e199, 2021. DOI: https://doi.org/10.24016/2021.v7.199.

Disponible en: https://revistainteracciones.com/index.php/rin/article/view/199. Accedido el: 8 ago. 2021.

SUÁREZ, J. et al. Percepción de los estudiantes sobre adaptaciones virtuales en cursos de anatomía humana por la contingencia SARS-CoV-2. Academia y Virtualidad, Nueva Granada, v. 14, n. 1, p. 151-168, 2021. DOI: https://doi.org/10.18359/ravi.5275.

Disponible en: https://revistas.unimilitar.edu.co/index.php/ravi/article/view/5275. Accedido el: 6 ago. 2021.

TOQUERO, C. Challenges and opportunities for higher education amid the Covid-19 pandemic: The Philippine Context. Pedagogical Research, Tahiti, v. 5, n. 4, em0063, 2020. DOI: https://doi.org/10.29333/pr/7947. Disponible en:

https://www.pedagogicalresearch.com/article/challenges-and-opportunities-for-highereducation-amid-the-covid-19-pandemic-the-philippine-context-7947. Accedido el: 6 ago. 2021.

VIZOSO, C.; ARIAS, O. Resiliencia, optimismo y burnout académico en estudiantes universitarios. European Journal of Education and Psychology, Santiago de Chile, v. 11, n. 1, p. 47-59, 2018. DOI: https://doi.org/10.30552/ejep.v11i1.185. Disponible en: https://revistas.uautonoma.cl/index.php/ejep/article/view/984. Accedido el: 6 ago. 2021.

Educ. Form., Fortaleza, v. 7, n. 1, e6759, jan./abr. 2022

DOI: https://doi.org/10.25053/redufor.v7i1.6759

https://revistas.uece.br/index.php/redufor/index 
YOUSSEF, F. Medical student stress, burnout and depression in Trinidad and Tobago. Academic Psychiatry, Berlín, v. 40, p. 69-75, 2016. DOl: https://doi.org/10.1007/s40596015-0468-9. Disponible en: https://link.springer.com/article/10.1007/s40596-015-0468-9. Accedido el: 6 ago. 2021.

\footnotetext{
Edwin Gustavo Estrada Araoz, Universidad Nacional Amazónica de Madre de Dios

i- https://orcid.org/0000-0003-4159-934X

Doctor en Educación, maestro en Administración de la Educación y Psicología Educativa, egresado de la Maestría en Políticas y Gestión de la Educación. Docente de pregrado en la Universidad Nacional Amazónica de Madre de Dios y en la Institución Educativa Almirante Miguel Grau Seminario. Contribución de autoría: Concepción, diseño de investigación, recolección de datos, análisis e interpretación de datos y revisión final del artículo.

E-mail: edwin5721@outlook.com
}

\begin{abstract}
Néstor Antonio Gallegos Ramos, Universidad Nacional Amazónica de Madre de Dios
ii(-)https://orcid.org/0000-0003-1436-9207

Doctor en Economía y Medio Ambiente, maestro en Ciencias de la Educación, ingeniero estatístico, especialista en Procesamiento y Análisis de Datos. Professor nombrado de la Universidad Nacional Amazónica de Madre de Dios, en la carrera profesional de Ingeniería de Sistemas.

Contribución de autoría: Metodología, recopilación, análisis e interpretación de datos y revisión de la última versión del manuscrito.

E-mail: ngallegor@gmail.com
\end{abstract}

Editora responsable: Lia Machado Fiuza Fialho Revisoras ad hoc: Mercedes Cathcart y Juana Robaina

\title{
Cómo citar este artículo (ABNT):
}

ESTRADA ARAOZ, Edwin Gustavo; GALLEGOS RAMOS, Néstor Antonio. Cansancio emocional en estudiantes universitarios peruanos en el contexto de la pandemia de Covid-19. Educ. Form., Fortaleza, v. 7, n. 1, e6759, 2022. Disponible en: https://revistas.uece.br/index.php/redufor/article/view/6759

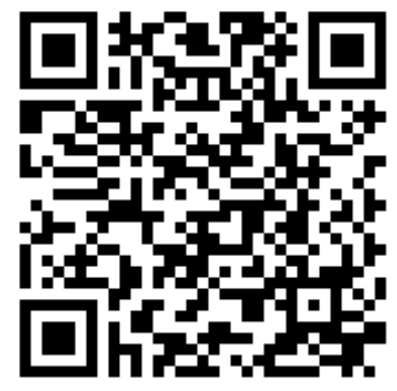

Recibido el 9 de agosto de 2021.

Aceptado el 17 de septiembre de 2021.

Publicado el 1 de enero de 2022.

Educ. Form., Fortaleza, v. 7, n. 1, e6759, jan./abr. 2022

DOI: https://doi.org/10.25053/redufor.v7i1.6759

https://revistas.uece.br/index.php/redufor/index 